\title{
A Sliding-rod Variable-strain Model for Concentric Tube Robots
}

\author{
Federico Renda ${ }^{1}$, Conor Messer ${ }^{2}$, Frederic Boyer ${ }^{3}$
}

\begin{abstract}
In this work, the Piecewise Variable-strain (PVS) approach is applied to the case of Concentric Tube Robots (CTRs) and extended to include the tubes' sliding motion. In particular, the currently accepted continuous Cosserat rod model is discretized onto a finite set of strain basis functions. At the same time, the insertion and rotation motions of the tubes are included as generalized coordinates instead of boundary kinematic conditions. Doing so, we obtain a minimum set of closed-form algebraic equations that can be solved not only for the shape variables but also for the actuation forces and torques for the first time. This new approach opens the way to torquecontrolled CTRs, which is poised to enhance elastic stability and improve interaction forces' control at the end-effector.
\end{abstract}

\section{INTRODUCTION}

Continuum robots can be considered a class of soft manipulators, particularly suited for Minimally Invasive Surgery (MIS) [1]. One of the most promising continuum robotic systems developed so far is the Concentric Tube Robot (CTR), a collection of nested millimeters tubes whose elastic interaction is used to control the system's overall shape and the iteration force at the end-effector for surgical intervention [2]. CTRs have demonstrated promising results in a variety of MIS applications. For a thorough survey of the clinical applications of CTRs, see [3] and the references therein.

The development of CTRs has been made possible thanks to rapid advancement in modeling these kinds of complex non-linear systems. These research efforts have converged on a select type of Cosserat rods model with a particular kinematic structure representing the internal tubes' additional rotational motion [4], [5]. Although this Cosserat approach has been improved over the years to include tubes' clearance, friction [6], and inertial dynamics [7], it has been essentially maintained as initially proposed [8]. Here, we refer to it as the standard CTR model, and we briefly recall it in section II.

On the other hand, a general soft robot is composed of flexible and rigid elements arranged in a parallel or serial fashion and capable of locomoting in the environment. A novel coordinates system has been proposed to model these kinds of systems, which discretizes the continuous Cosserat model of the flexible components onto a finite set of strain basis functions [9], [10]. This Piecewise Variable-strain (PVS) approach is a

Manuscript received: October, 22, 2020; Revised , , 2021; Accepted , , .

This work was supported by the Khalifa University of Science and Technology under Award No. FSU-2018-08, RC2-2018-HEIC, CIRA-2020-074 and ADEK Award for Research Excellence (AARE-2018-105).

1 Khalifa University Center for Autonomous Robotic Systems and the Healthcare Engineering Innovation Center, Khalifa University of Science and Technology, Abu Dhabi, UAE. Corresponding author federico.renda@ku. ac. ae

2 Fulbright Student Researcher placed at Khalifa University of Science and Technology, Abu Dhabi, UAE conorsmesseregmail. com

3 LS2N lab, Institut Mines Telecom Atlantique, 44307 Nantes, France. frederic.boyerdimt-atlantique. fr

Digital Object Identifier (DOI): see top of this page. generalization of traditional robotics' geometric model [11] to the case of highly flexible or soft robots [12]. Thus, it provides the theoretical framework for applying traditional control strategies to the field of soft robots. One of the aims of the present work is to apply the PVS approach to CTR systems. In this way, we further extend the general PVS approach to this essential soft robotic technology. At the same time, we open new prospects for the control design of CTRs. In particular, the PVS model provides the equilibrium equations as a minimum set of closed-form algebraic equations, which can be easier to handle for control and design optimization.

Furthermore, in this work, we tackle one of the main limitations of the standard CTR model concerned with the sliding structure problem, also known as the spaghetti problem [13]. In the standard model, the tubes' insertion is prescribed once and rigidly defines the system's sections' domains. This prevents to fully model the sliding motion of the tubes. In fact, the recent extension to dynamics assumes negligible insertion velocity and acceleration [7]. Here we relax this assumption, although still in a static setting, which allows calculating the actuation forces and torques required for the equilibrium. To the authors' knowledge, it is the first time that such a model is proposed for CTRs. The spaghetti problem appears in the planar models of animal locomotion [14], structural stability [15], and finite element analysis [16]. Thus, to the authors' knowledge, it is also the first time the spaghetti problem is tackled in three dimensions and with an additional relative rotation.

\section{MODEL PRELIMINARIES}

Before applying the PVS approach to solve the CTR equilibrium, we revise the standard CTR model [4], [5] using geometric notation. For simplicity, we will present the case of fully overlapping tubes here. Note that additional details about the CTR kinematics will be provided for the more general cases analyzed in section III. Furthermore, a new kinematic model that includes the sliding and rotation of the the bases of the tubes as generalized coordinates of the system will be introduced in section IV.

\section{A. Kinematics}

Each tube of a concentric tube system can be modeled as a Cosserat rod, a continuous set of rigid cross sections of infinitesimal thickness along a material curvilinear abscissa $X \in[0, L]$ where $L$ is the total length of the rod. Identifying each rigid cross-section with the moving frame rigidly attached to it, the configuration of the tube is completely defined by a curve $\boldsymbol{g}(\cdot): X \mapsto \boldsymbol{g}(X) \in S E(3)$ represented by the homogeneous matrix:

$$
\boldsymbol{g}=\left(\begin{array}{cc}
\mathbf{R} & \mathbf{r} \\
\mathbf{0}^{T} & 1
\end{array}\right)
$$


where $\mathbf{r}(X) \in \mathbb{R}^{3}$ is the position vector of the origin of the moving frame and $\mathbf{R}(X) \in S O(3)$ is the rotation matrix representing its orientation with respect to the spatial frame.

The high aspect ratio and material property of the conventional CTR allow assuming inextensibility and shearless deformation (Kirchhoff-Love kinematics). Furthermore, the tubes being concentric, their centerline must be the same at any configuration. Then, it follows that an inner tube differs from its outer tube only by a rotation around the tangent vector to the centerline [5], defined by:

$$
\boldsymbol{g}_{\theta}(X)=\left(\begin{array}{cccc}
1 & 0 & 0 & 0 \\
0 & \cos (\theta) & -\sin (\theta) & 0 \\
0 & \sin (\theta) & \cos (\theta) & 0 \\
0 & 0 & 0 & 1
\end{array}\right)
$$

where $\theta(X)$ is the rotation angle and we have assumed a local coordinate frame as shown in Figure 1. Thus, for a tube $j$ we obtain:

$$
\begin{aligned}
& \boldsymbol{g}_{1}(X)=\boldsymbol{g}_{1}(X) \\
& \boldsymbol{g}_{j}(X)=\boldsymbol{g}_{j-1}(X) \boldsymbol{g}_{\theta_{j}}(X),(j=2, \ldots, N)
\end{aligned}
$$

where $N$ is the total number of tubes. Note that in this model, the concentric tubes share the same material abscissa $X$.

Let's obtain the time $\left(\partial / \partial t={ }^{\prime}\right)$ and space $\left(\partial / \partial X={ }^{\prime}\right)$ derivative of the tubes configuration $\boldsymbol{g}$ with the Lie algebra $\mathfrak{s e}(3)$. Starting with tube 1 we get:

$$
\begin{aligned}
& \left(\boldsymbol{g}_{1}^{-1}(X) \dot{\boldsymbol{g}}_{1}(X)\right)^{\vee}=\boldsymbol{\eta}_{1}(X)=\left(\mathbf{w}_{1}^{T}, \mathbf{v}_{1}^{T}\right)^{T} \in \mathbb{R}^{6} \\
& \left(\boldsymbol{g}_{1}^{-1}(X) \boldsymbol{g}_{1}^{\prime}(X)\right)^{\vee}=\boldsymbol{\xi}_{1}(X)=\left(\mathbf{k}_{1}^{T}, \mathbf{u}_{1}^{T}\right)^{T} \in \mathbb{R}^{6} .
\end{aligned}
$$

where $\mathbf{v}(X), \mathbf{u}(X) \in \mathbb{R}^{3}$ represent the linear strains and velocity respectively, while $\mathbf{w}(X), \mathbf{k}(X) \in \mathbb{R}^{3}$ are the angular strains and velocity respectively. All the quantities are defined in the local frame at $X$. To indicate the angular strain in the reference stress-free configuration, we use the notation $\mathbf{k}^{*}$. Finally, the superscript $\vee$ indicates the isomorphism between the Lie algebra $\mathfrak{s e}(3)$ and $\mathbb{R}^{6}$ [11] ( $\wedge$ will be used in the opposite direction).

Equating the mixed partial derivatives of the tube configuration, we obtain a compatibility equation between the velocity and strain twists.

$$
\frac{\partial}{\partial t} \boldsymbol{g}_{1}^{\prime}=\frac{\partial}{\partial X} \dot{\boldsymbol{g}}_{1} \Longrightarrow \boldsymbol{\eta}_{1}^{\prime}=\dot{\boldsymbol{\xi}}_{1}-\operatorname{ad}_{\boldsymbol{\xi}_{1}} \boldsymbol{\eta}_{1}
$$

As shown in [9], integrating (6) with respect to space yields the following useful relation:

$$
\boldsymbol{\eta}_{1}(X)=\operatorname{Ad}_{\boldsymbol{g}_{1}(X)}^{-1} \int_{0}^{X} \operatorname{Ad}_{\boldsymbol{g}_{1}} \dot{\boldsymbol{\xi}}_{1} d s,
$$

where the operator Ad is the Adjoint map in $S E(3)$ defined in Appendix A.

For what concerns the inner tubes, first we define the derivatives of the relative rotation $\boldsymbol{g}_{\theta}$.

$$
\begin{aligned}
& \left(\boldsymbol{g}_{\theta}^{-1}(X) \dot{\boldsymbol{g}}_{\theta}(X)\right)^{\vee}=\boldsymbol{\eta}_{\theta}(X)=\left[\begin{array}{llllll}
\dot{\theta}(X) & 0 & 0 & 0 & 0 & 0
\end{array}\right]^{T} \\
& \left(\boldsymbol{g}_{\theta}^{-1}(X) \boldsymbol{g}_{\theta}^{\prime}(X)\right)^{\vee}=\boldsymbol{\xi}_{\theta}(X)=\left[\begin{array}{llllll}
\theta^{\prime}(X) & 0 & 0 & 0 & 0 & 0
\end{array}\right]^{T} .
\end{aligned}
$$

The same procedure that led to equation (7), in this case yields:

$$
\boldsymbol{\eta}_{\theta}(X)=\int_{0}^{X} \dot{\boldsymbol{\xi}}_{\theta} d s,
$$

Finally, the chain rule together with (4), (5), (8), and (9) yields, for a general tube $j$ :

$$
\begin{aligned}
& \left(\boldsymbol{g}_{j}^{-1} \dot{\boldsymbol{g}}_{j}\right)^{\vee}=\boldsymbol{\eta}_{j}(X)=\prod_{i=j}^{2} \operatorname{Ad}_{\boldsymbol{g}_{\theta_{i}}}^{-1} \boldsymbol{\eta}_{1}(X)+\sum_{i=2}^{j} \boldsymbol{\eta}_{\theta_{i}}(X) \\
& \left(\boldsymbol{g}_{j}^{-1} \boldsymbol{g}_{j}^{\prime}\right)^{\vee}=\boldsymbol{\xi}_{j}(X)=\prod_{i=j}^{2} \operatorname{Ad}_{\boldsymbol{g}_{\theta_{i}}}^{-1} \boldsymbol{\xi}_{1}(X)+\sum_{i=2}^{j} \boldsymbol{\xi}_{\theta_{i}}(X) .
\end{aligned}
$$

\section{B. Statics}

In this work, we focus our attention on the static equilibrium of concentric tubes with no external applied force (except for the actuation in section IV) to get more neat results. However, it should be noted that there are no particular impediments that would prevent extending the approach to the general dynamic case, as recently done for the standard CTR model in [7]. This extension will be targeted in future work.

The static equilibrium of a Cosserat rod in a concentric tube setting can be derived from the general equilibrium equation (see [17] for a derivation) with the addition of the constraints wrenches due to the concentricity constraint. Thus, for a tube $j$ we obtain:

$$
\mathcal{F}_{i_{j}}^{\prime}+\operatorname{ad}_{\boldsymbol{\xi}_{j}}^{*} \mathcal{F}_{i_{j}}+\overline{\mathcal{F}}_{\lambda_{j}}=\mathbf{0},(j=1, \ldots, N),
$$

where $\mathcal{F}_{i_{j}}(X)=\left(\mathbf{M}_{i_{j}}^{T}(X), \mathbf{N}_{i_{j}}^{T}(X)\right)^{T} \in \mathbb{R}^{6}$ is the wrench of internal moment and force, $\overline{\mathcal{F}}_{\lambda_{j}}(X) \in \mathbb{R}^{6}$ is the wrench of distributed constraint force, and $\mathrm{ad}^{*}$ is the co-adjoint map in $S E(3)$ defined in Appendix A. Note that the value of the internal force $\mathbf{N}_{i_{j}}$ is unknown due to the inextensibility and shearless constraints. On the other hand, the internal moment's value can be computed from a constitutive law that we assume linear for simplicity.

$$
\mathbf{M}_{i_{j}}(X)=\mathbf{\Sigma}_{j}\left(\mathbf{k}_{j}(X)-\mathbf{k}_{j}^{*}(X)\right)
$$

where $\boldsymbol{\Sigma}=\operatorname{diag}(G I, E J, E J) \in \mathbb{R}^{3 \times 3}$ is the elasticity matrix, $E$ is the Young modulus, $G$ the shear modulus, and $I, J$ are, respectively, the polar and bending second moment of area of the circular cross-section. For what concerns the constraint contact force, although its value is also unknown, its basis can be obtained from the virtual constraints equations that enforce the concentricity constraint. These equations can be written in the form

$$
\begin{aligned}
\mathbf{j}_{j} \cdot\left(\delta \mathbf{r}_{j}(X)-\delta \mathbf{r}_{j-1}(X)\right) & =0,(j=2, \ldots, N) \\
\mathbf{k}_{j} \cdot\left(\delta \mathbf{r}_{j}(X)-\delta \mathbf{r}_{j-1}(X)\right) & =0,(j=2, \ldots, N),
\end{aligned}
$$

where $\mathbf{j}_{j}$ and $\mathbf{k}_{j}$ point in the $y-$, and $z$-axis directions of tube $j$ at $X$ (see Fig. 1). Equation (15) says that the distributed constraint force is equal and opposite between two consecutive tubes and that it takes the form:

$$
\begin{aligned}
\overline{\mathcal{F}}_{\lambda_{j}}(X)= & {\left[\begin{array}{llllll}
0 & 0 & 0 & 0 & \lambda_{y_{j}}(X) & \lambda_{z_{j}}(X)
\end{array}\right]^{T}-} \\
& \operatorname{Ad}_{\boldsymbol{g}_{\theta_{j+1}}^{*}}^{*}\left[\begin{array}{llllll}
0 & 0 & 0 & 0 & \lambda_{y_{j+1}}(X) \lambda_{z_{j+1}}(X)
\end{array}\right]^{T},
\end{aligned}
$$


where $\lambda_{y}(X)$ and $\lambda_{z}(X)$ are Lagrange multipliers, and $\mathrm{Ad}^{*}$ is the co-Adjoint map in SE(3) defined in Appendix A.

The static equilibrium of a collection of tubes imposes the following boundary conditions on equation (13) [5].

$$
\sum_{j=1}^{N} \mathbf{M}_{i_{j}}\left(\bar{X}^{-}\right)=\sum_{j=1}^{N} \mathbf{M}_{i_{j}}\left(\bar{X}^{+}\right) .
$$

Note that (17) also holds at discontinuity points $\bar{X}$ where the tubes stop to overlap (in that case, the upper limit of the sum on the RHS will be smaller). Furthermore, since the concentric tubes can not transmit axial torque to one another, the following condition holds for each tube [5].

$$
\mathbf{i} \cdot \mathbf{M}_{i}\left(\bar{X}^{-}\right)=\mathbf{i} \cdot \mathbf{M}_{i}\left(\bar{X}^{+}\right),
$$

where $\mathbf{i}$ points in the direction tangent to the midline.

\section{VARIABLE-STRAIN CTR MODEL}

In [9], [10], a novel variable-strain approach has been presented to model soft manipulators driven by tendons and pneumatic chambers. This section aims to apply this technique to CTRs to obtain a minimum set of closed-form equations describing the system equilibrium.

According to the PVS approach, the configuration $\boldsymbol{g}_{j}$ of the collection of tubes is represented by the strain fields $\boldsymbol{\xi}_{1}$ and $\boldsymbol{\xi}_{\theta_{j}}$. In particular, the infinite dimensional strain fields are discretized on a finite set of basis functions as follows.

$$
\begin{aligned}
\boldsymbol{\xi}_{1}(X) & =\boldsymbol{B}_{1}(X) \boldsymbol{p}_{1}+\overline{\boldsymbol{\xi}}, \\
\boldsymbol{\xi}_{\theta_{j}}(X) & =\boldsymbol{B}_{\theta_{j}}(X) \boldsymbol{p}_{\theta_{j}},
\end{aligned}
$$

where $\boldsymbol{B}_{1}(X) \in \mathbb{R}^{6 \times n 1}$ and $\boldsymbol{B}_{\theta_{j}}(X) \in \mathbb{R}^{6 \times n \theta_{j}}$ are matrix functions whose columns form the basis for the strain field $\boldsymbol{\xi}_{1}$ and $\boldsymbol{\xi}_{\theta_{j}}$, respectively, while $\boldsymbol{p}_{1} \in \mathbb{R}^{n 1}$ and $\boldsymbol{p}_{\theta_{j}} \in \mathbb{R}^{n \theta_{j}}$ are the vectors of coordinates. Note that, due to the assumptions made in section II, $\overline{\boldsymbol{\xi}}$ is equal to $\left[\begin{array}{llllll}0 & 0 & 0 & 1 & 0 & 0\end{array}\right]$, while the last three rows of $\boldsymbol{B}_{1}(X)$ and the last five rows of $\boldsymbol{B}_{\theta_{j}}(X)$ are all equal to zero. Then, the set of generalized coordinates become $\boldsymbol{q}=\left(\boldsymbol{p}_{1}, \boldsymbol{p}_{\theta_{2}}, \ldots, \boldsymbol{p}_{\theta_{N}}\right)$, and the configuration $\boldsymbol{g}_{j}(\boldsymbol{q})$ can be reconstructed through the integration of equations (5), (9) and the recursive formula given by (3).

The differential relation between configuration and generalized coordinates is obtained by replacing equations (19), (20) in the velocity equations (7), (10), which yields

$$
\begin{aligned}
\boldsymbol{\eta}_{1}(X) & =\left[\operatorname{Ad}_{\boldsymbol{g}_{1}}^{-1} \int_{0}^{X} \operatorname{Ad}_{\boldsymbol{g}_{1}} \boldsymbol{B}_{1} d s\right] \dot{\boldsymbol{p}}_{1}=\boldsymbol{S}_{1}(X) \dot{\boldsymbol{p}}_{1}, \\
\boldsymbol{\eta}_{\theta_{j}}(X) & =\left[\int_{0}^{X} \boldsymbol{B}_{\theta_{j}} d s\right] \dot{\boldsymbol{p}}_{\theta_{j}}=\boldsymbol{J}_{\theta_{j}}(X) \dot{\boldsymbol{p}}_{\theta_{j}},
\end{aligned}
$$

and, in turn, replacing the result in the velocity equation (11). Finally, we obtain:

$$
\begin{aligned}
\boldsymbol{\eta}_{j}(X) & =\left[\prod_{i=j}^{2} \operatorname{Ad}_{\boldsymbol{g}_{\theta_{i}}^{-1}}^{-1} \boldsymbol{S}_{1}(X)\right] \dot{\boldsymbol{p}}_{1}+\sum_{i=2}^{j} \boldsymbol{J}_{\theta_{i}}(X) \dot{\boldsymbol{p}}_{\theta_{i}} \\
& =\boldsymbol{J}_{j}(X) \dot{\boldsymbol{p}}_{1}+\sum_{i=2}^{j} \boldsymbol{J}_{\theta_{i}}(X) \dot{\boldsymbol{p}}_{\theta_{i}} .
\end{aligned}
$$

Note that $\boldsymbol{J}_{\theta_{i}}(X)$ is an analytical function that can be computed offline given the choice of basis $\boldsymbol{B}_{\theta_{i}}$. The differential equation (23) provides the required Jacobians to project the static equilibrium (13) by d'Alembert's principle. In particular, equation (13) is projected with $\int_{0}^{L} \boldsymbol{J}_{j}^{T} d X$ and $\int_{0}^{L} \boldsymbol{J}_{\theta_{i}}^{T} d X$ for all $j$ and $i$. The results are then summed over all $j$ from 1 to $N$ to obtain as much algebraic equilibrium equation as the dimension of $\boldsymbol{q}$. This procedure is shown in detail for the case of two fully overlapping tubes in the next section. The general case of multiple non-overlapping tubes with straight actuation, as shown in Figure 3, will be illustrated in the following.

\section{A. Single overlapping sections}

Let us consider the case of two fully overlapping tubes. Applying the d'Alembert's principle as described above yields:

$$
\begin{array}{r}
\sum_{j=1}^{2} \int_{0}^{L} \boldsymbol{J}_{j}^{T}\left(\mathcal{F}_{i_{j}}^{\prime}+\operatorname{ad}_{\boldsymbol{\xi}_{j}}^{*} \mathcal{F}_{i_{j}}+\overline{\mathcal{F}}_{\lambda_{j}}\right) d X=\mathbf{0} \\
\int_{0}^{L} \boldsymbol{J}_{\theta_{2}}^{T}\left(\mathcal{F}_{i_{2}}^{\prime}+\operatorname{ad}_{\boldsymbol{\xi}_{2}}^{*} \mathcal{F}_{i_{2}}+\overline{\mathcal{F}}_{\lambda_{2}}\right) d X=\mathbf{0} .
\end{array}
$$

Considering the form of the constraint force (16) and the identity $\mathcal{F}_{i_{j}}^{\prime}+\operatorname{ad}_{\boldsymbol{\xi}_{j}}^{*} \mathcal{F}_{i_{j}}=\operatorname{Ad}_{\boldsymbol{g}_{j}^{-1}}^{*}\left(\operatorname{Ad}_{\boldsymbol{g}_{j}}^{*} \mathcal{F}_{i_{j}}\right)^{\prime}$, we get (note that $\left.\operatorname{Ad}_{\boldsymbol{g}}^{T}=\operatorname{Ad}_{\boldsymbol{g}^{-1}}^{*}\right)$ :

$$
\begin{aligned}
\sum_{j=1}^{2} \int_{0}^{L}\left(\int_{0}^{X} \boldsymbol{B}_{1}^{T} \operatorname{Ad}_{\boldsymbol{g}_{1}}^{T} d s\right)\left(\operatorname{Ad}_{\boldsymbol{g}_{j}} \mathcal{F}_{i_{j}}\right)^{\prime} d X & =\mathbf{0} \\
\int_{0}^{L}\left(\int_{0}^{X} \boldsymbol{B}_{\theta_{2}}^{T} d s\right) \operatorname{Ad}_{\boldsymbol{g}_{2}^{-1}}^{*}\left(\operatorname{Ad}_{\boldsymbol{g}_{2}} \mathcal{F}_{i_{2}}\right)^{\prime} d X & =\mathbf{0} .
\end{aligned}
$$

Finally, integrating by part we obtain:

$$
\begin{aligned}
& \int_{0}^{L} \boldsymbol{B}_{1}^{T}\left(\mathcal{F}_{i_{1}}+\operatorname{Ad}_{\boldsymbol{g}_{\theta_{2}}}^{*} \mathcal{F}_{i_{2}}\right) d X=\mathbf{0} \\
& \int_{0}^{L} \boldsymbol{B}_{\theta_{2}}^{T} \mathcal{F}_{i_{2}} d X=\int_{0}^{L} \boldsymbol{J}_{\theta_{2}}^{T} \operatorname{ad}_{\boldsymbol{\xi}_{1}}^{*} \operatorname{Ad}_{\boldsymbol{g}_{\theta_{2}}}^{*} \mathcal{F}_{i_{2}} d X,
\end{aligned}
$$

where we have used the strain equation (12), the identity $\boldsymbol{J}_{\theta_{2}}^{T} \operatorname{ad}_{\boldsymbol{\xi}_{\theta_{2}}}^{*} \mathcal{F}_{i_{2}}=\mathbf{0}$ and the boundary conditions (17), (18) at $L$. Note that, since the generalized coordinates $\boldsymbol{q}$ are independent, the unknown constraint forces $\overline{\mathcal{F}}_{\lambda_{j}}$ cancel out as expected.

Equation (26) can be solved numerically for the unknowns $\boldsymbol{p}_{1}$ and $\boldsymbol{p}_{\theta_{2}}$ that appear in $\mathcal{F}_{i_{j}}, \boldsymbol{\xi}_{1}$, and $\boldsymbol{g}_{\theta_{2}}$ through equations (19), (20), (14), and (9). Note that the integration of (9) becomes very simple in this case. Specifically, the rotation angle $\theta$ of (2) is obtained by:

$$
\theta_{2}(X)=\alpha_{2}+\left(\boldsymbol{J}_{\theta_{2}}(X) \boldsymbol{p}_{\theta_{2}}\right)_{x},
$$

where ()$_{x}$ extracts the first rotational element of a vector and $\alpha_{2}$ is the rotation of the tube imposed at the base by the actuators.

\section{Comparison with analytic torsionless case}

As a first test to check the validity of the proposed model, we consider here the case of two torsionless, fully overlapping tubes with three-dimensional deformation. The torsionless approximation was used at the beginning of the CTR development, and analytic solutions are available for the 
constant curvature approximation [18], [19], [20] as well as the general unconstrained curvature case [21].

Assuming no torsion, the equilibrium equation (26) becomes:

$$
\int_{0}^{L} \boldsymbol{B}_{1}^{T}\left(\mathcal{F}_{i_{1}}+\mathcal{F}_{i_{2}}\right) d X=\mathbf{0}
$$

with $\theta_{2}=0$ and $\boldsymbol{\xi}_{1}=\boldsymbol{\xi}_{2}=\boldsymbol{\xi}$. To reproduce the available analytic solution with unconstrained curvature [21], we select the following basis for the strain $\boldsymbol{\xi}$.

$$
\boldsymbol{B}_{1}^{T}(X)=\left[\begin{array}{cccccc}
0 & \left(\mathbf{k}_{1}^{*}\right)_{y}(X) & 0 & 0 & 0 & 0 \\
0 & \left(\mathbf{k}_{2}^{*}\right)_{y}(X) & 0 & 0 & 0 & 0 \\
0 & 0 & \left(\mathbf{k}_{1}^{*}\right)_{z}(X) & 0 & 0 & 0 \\
0 & 0 & \left(\mathbf{k}_{2}^{*}\right)_{z}(X) & 0 & 0 & 0
\end{array}\right],
$$

where $\mathbf{k}_{j}^{*}$ is the reference strain of tube $j$. Thus, $n_{1}=4$ and the generalized coordinates $\boldsymbol{p}_{1}=\boldsymbol{q}=\left[\begin{array}{llll}q_{1} & q_{2} & q_{3} & q_{4}\end{array}\right]^{T}$ represent the amount of reference curvatures making up the equilibrium configuration. Rearranging (28), we can write:

$$
\begin{gathered}
\left(\int_{0}^{L}\left[\begin{array}{cc}
\left(\mathbf{k}_{1}^{*}\right)_{y}^{2} & \left(\mathbf{k}_{1}^{*}\right)_{y}\left(\mathbf{k}_{2}^{*}\right)_{y} \\
\left(\mathbf{k}_{2}^{*}\right)_{y}\left(\mathbf{k}_{1}^{*}\right)_{y} & \left(\mathbf{k}_{2}^{*}\right)_{y}^{2}
\end{array}\right] d X\right)\left[\begin{array}{c}
q_{1} \\
q_{2}
\end{array}\right]= \\
\frac{1}{\left(E J_{1}+E J_{2}\right)} \int_{0}^{L}\left[\begin{array}{l}
E J_{1}\left(\mathbf{k}_{1}^{*}\right)_{y}^{2}+E J_{2}\left(\mathbf{k}_{1}^{*}\right)_{y}\left(\mathbf{k}_{2}^{*}\right)_{y} \\
E J_{1}\left(\mathbf{k}_{2}^{*}\right)_{y}\left(\mathbf{k}_{1}^{*}\right)_{y}+E J_{2}\left(\mathbf{k}_{2}^{*}\right)_{y}^{2}
\end{array}\right] d X .
\end{gathered}
$$

A similar equation can be obtained for $q_{3}$ and $q_{4}$. Solving for $\boldsymbol{q}$, we get:

$$
\boldsymbol{q}=\frac{1}{\left(E J_{1}+E J_{2}\right)}\left[\begin{array}{llll}
E J_{1} & E J_{2} & E J_{1} & E J_{2}
\end{array}\right]^{T},
$$

which correctly corresponds to the analytic solution $\mathbf{k}=$ $\left(\boldsymbol{\Sigma}_{1}+\boldsymbol{\Sigma}_{2}\right)^{-1}\left(\boldsymbol{\Sigma}_{1} \mathbf{k}_{1}^{*}+\boldsymbol{\Sigma}_{2} \mathbf{k}_{2}^{*}\right)$.

\section{B. Multiple non-overlapping sections}

Let us now move to the case of multiple non-overlapping tubes. To approach this problem, we divide the system domain into piecewise variable-strain sections corresponding to the curvature discontinuity located at the end of every overlapping portion. Thus, the kinematic equation (3) is applicable locally at each overlapping portion and can be generalized as follows (see Figure 1):

$$
\begin{aligned}
\boldsymbol{g}_{j_{s}}(X) & =\left(\prod_{i=1}^{j-1} \boldsymbol{g}_{i}\left(L_{i}\right) \boldsymbol{g}_{\theta_{(i+1) s}}\left(L_{i}\right)\right) \boldsymbol{g}_{j}(X) \\
\boldsymbol{g}_{k_{s}}(X) & =\boldsymbol{g}_{(k-1)_{s}}(X) \boldsymbol{g}_{\theta_{k_{s}}}(X),(k=j+1, \ldots, N),
\end{aligned}
$$

where $\boldsymbol{g}_{j_{s}}$ indicates the configuration of tube $j$ with respect to the spatial frame, while $\boldsymbol{g}_{j}$ represents the configuration with respect to the local overlapping portion. $L_{i}$ is the length of tube $i$, which corresponds to the end of an overlapping portion. $\boldsymbol{g}_{\theta_{j_{s}}}(X)$ is the total rotation between tube $j$ and tube $j-1$ at $X$, which is obtained through the product of the partial rotation of each overlapping portion preceding $X$. For example, consider the total rotation between tube $j$ and tube $j-1$ at $X$ located on the third overlapping section, then $\boldsymbol{g}_{\theta_{j_{s}}}(X)=\boldsymbol{g}_{\theta_{j_{1}}}\left(L_{1}\right) \boldsymbol{g}_{\theta_{j_{2}}}\left(L_{2}\right) \boldsymbol{g}_{\theta_{j_{3}}}(X)$.

A procedure similar to the single overlapping sections case leads to the equilibrium equations for general multiple nonoverlapping tubes in the generalized variable-strain coordinates.

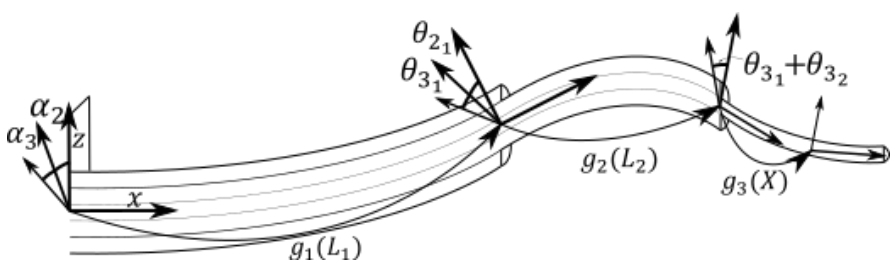

Fig. 1: Kinematics of multiple non-overlapping sections. For each overlapping portion the kinematic equation (3) applies.

For example, consider the case of three non-overlapping tubes, then we obtain:

$$
\begin{aligned}
& \int_{0}^{L_{1}} \boldsymbol{B}_{1}^{T}\left(\mathcal{F}_{i_{1}}+\operatorname{Ad}_{\boldsymbol{g}_{\theta_{2}}}^{*}\left(\mathcal{F}_{i_{2}}+\operatorname{Ad}_{\boldsymbol{g}_{\theta_{3}}}^{*} \mathcal{F}_{i_{3}}\right)\right) d X=\mathbf{0} \\
& \int_{0}^{L_{1}} \boldsymbol{B}_{\theta_{2_{1}}}^{T}\left(\mathcal{F}_{i_{2}}+\mathcal{F}_{i_{3}}\right) d X= \\
& \int_{0}^{L_{1}} \boldsymbol{J}_{\theta_{2_{1}}}^{T} \operatorname{ad}_{\boldsymbol{\xi}_{1}}^{*} \operatorname{Ad}_{\boldsymbol{g}_{\theta_{2}}}^{*}\left(\mathcal{F}_{i_{2}}+\operatorname{Ad}_{\boldsymbol{g}_{\theta_{3}}}^{*} \mathcal{F}_{i_{3}}\right) d X \\
& \int_{0}^{L_{1}} \boldsymbol{B}_{\theta_{3_{1}}}^{T} \mathcal{F}_{i_{3}}=\int_{0}^{L_{1}} \boldsymbol{J}_{\theta_{3_{1}}}^{T} \operatorname{ad}_{\boldsymbol{\xi}_{1}}^{*} \operatorname{Ad}_{\boldsymbol{g}_{\theta_{2}} \boldsymbol{g}_{\theta_{3_{s}}}}^{*} \mathcal{F}_{i_{3}} d X+ \\
& \boldsymbol{J}_{\theta_{3_{1}}}^{T}\left(L_{1}\right) \int_{L_{1}}^{L_{2}} \operatorname{ad}_{\boldsymbol{\xi}_{2}}^{*} \operatorname{Ad}_{\boldsymbol{g}_{\theta_{3}}}^{*} \mathcal{F}_{i_{3}} d X \\
& \int_{L_{1}}^{L_{2}} \boldsymbol{B}_{2}^{T}\left(\mathcal{F}_{i_{2}}+\operatorname{Ad}_{\boldsymbol{g}_{\theta_{3}}}^{*} \mathcal{F}_{i_{3}}\right) d X=\mathbf{0} \\
& \int_{L_{1}}^{L_{2}} \boldsymbol{B}_{\theta_{3_{2}}}^{T} \mathcal{F}_{i_{3}} d X=\int_{L_{1}}^{L_{2}} \boldsymbol{J}_{\theta_{3_{2}}}^{T} \operatorname{ad}_{\boldsymbol{\xi}_{2}}^{*} \operatorname{Ad}_{\boldsymbol{g}_{\theta_{3}}}^{*} \mathcal{F}_{i_{3}} d X \\
& \int_{L_{2}}^{L_{3}} \boldsymbol{B}_{3}^{T} \mathcal{F}_{i_{3}} d X=\mathbf{0} \text {. }
\end{aligned}
$$

As with (26), equation (33) can be solved numerically for the unknown $\boldsymbol{q}=\left(\boldsymbol{p}_{1}, \boldsymbol{p}_{\theta_{2_{1}}}, \boldsymbol{p}_{\theta_{3_{1}}}, \boldsymbol{p}_{2}, \boldsymbol{p}_{\theta_{3_{2}}}, \boldsymbol{p}_{3}\right)$.

\section{Comparison with simulation in literature}

The multiple overlapping tubes model is tested by comparing the equilibrium configuration obtained for a two tubes robot with a literature benchmark reported in [21]. Consider two tubes of length $140 \mathrm{~mm}$ (outer) and $200 \mathrm{~mm}$ (inner) with constant pre-curvature and physical properties reported in Table I. We seek for the equilibrium configuration when the inner tube is rotated by $\alpha_{2}=180^{\circ}$. The system domain is divided into two variable-strain sections. The generalized coordinates are $\boldsymbol{q}=\left(\boldsymbol{p}_{1}, \boldsymbol{p}_{\theta_{2}}, \boldsymbol{p}_{2}\right)$, and the equilibrium equations are a subset of (33). The resulting model takes the form of a nonlinear algebraic system of equations that can be solved numerically with the MATLAB ${ }^{\odot}$ fsolve function. We chose a linear nonhomogeneous base for the strains involved in the first section, i.e.: $\mathbf{k}_{1}$, and $\theta_{2}^{\prime}$. Then, we have

$$
\boldsymbol{B}_{1}=\left[\begin{array}{cccccc}
1 & X & 0 & 0 & 0 & 0 \\
0 & 0 & 1 & X & 0 & 0 \\
0 & 0 & 0 & 0 & 1 & X \\
0 & 0 & 0 & 0 & 0 & 0 \\
0 & 0 & 0 & 0 & 0 & 0 \\
0 & 0 & 0 & 0 & 0 & 0
\end{array}\right], \boldsymbol{B}_{\theta_{2}}=\left[\begin{array}{cc}
1 & X \\
0 & 0 \\
0 & 0 \\
0 & 0 \\
0 & 0 \\
0 & 0
\end{array}\right],
$$

For the second section, since it is unloaded, we use the reference strain $\mathbf{k}_{2}^{*}$ as a basis for the strain $\mathbf{k}_{2}$, which gives $\boldsymbol{p}_{2}=1$. 
TABLE I: Physical Properties of Tubes

\begin{tabular}{ccc}
\hline & Outer tube & Inner tube \\
\hline Young's Modulus $(\mathrm{GPa})$ & 58 & 58 \\
\hline Shear Modulus $(\mathrm{GPa})$ & 21.5 & 21.5 \\
\hline Inner diameter $(\mathrm{mm})$ & 2.01 & 0 \\
\hline Outer diameter $(\mathrm{mm})$ & 2.39 & 1.6 \\
\hline Reference curvature $\left(\mathrm{mm}^{-1}\right)$ & 0.0099 & 0.0138 \\
\hline
\end{tabular}

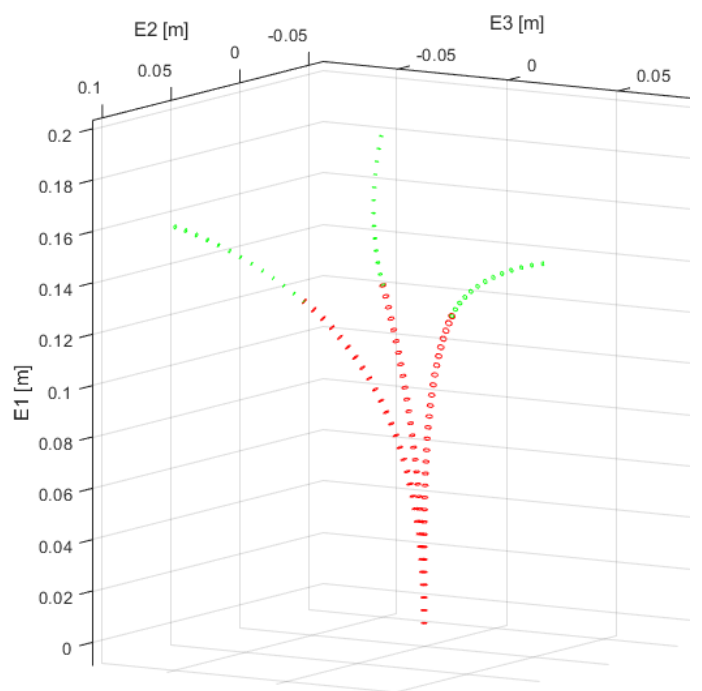

Fig. 2: Equilibrium configurations computed with the variable-strain model for two concentric tubes with constant pre-curvature. The inner tube is rotated by $180^{\circ}$. The result of the variable-strain model match well with the one off the standard CTR model [21].

As illustrated in [21], the CTR has three equilibrium configurations. The solution to which the solver converges depends on the initial guess. Figure 2 illustrates the equilibrium configurations obtained with the variable-strain model. A visual comparison shows a good match with the standard CTR model (Fig. 3 of [21]). Furthermore, the inner tube's rotation with respect to the outer tube at the end of the overlapping section is equal to $\theta_{2}\left(L_{1}\right)= \pm 85.5^{\circ}$, which is only $1.3 \%$ bigger than the value reported in $[21]\left( \pm 84.4^{\circ}\right)$.

\section{Complete CTR system}

The CTR systems considered so far only include the tubes' portions that follow the insertion orifice, assuming that the rotation input $\alpha$ was applied there (see equation (27)). However, a complete CTR system is also composed of a straight portion that precedes the insertion point and terminates with an actuation system responsible for applying the input angle and insertion motion. To include this part, we place the 0 of the system domain $X$ at the innermost tube base. Then, we divide the system domain into several sections corresponding to the different discontinuities. Those include a change in the number of overlapping tubes (as before), the insertion orifice, and, eventually, a reference strain jump. For instance, the straight portion is usually obtained by letting the tube be originally straight at the proximal end and curved toward the distal end. Finally, we constrain the straight portion by choosing only a torsional strain basis for these proximal sections. Note that this method applies to fully curved tubes as well, where external constraints enforce the straightness condition.
For example, let us consider the two tubes CTR system shown in Figure 3, where each tube has a straight proximal reference strain of length $L_{j_{s}}$ as indicated. There are five sections in this example indicated with an additional subscript in the following. Define $D_{1}, D_{2}$ to be the distance between the insertion orifice and the outer and inner tube base, respectively, and $\Delta D=D_{2}-D_{1}$. Then, the equilibrium equations for the generalized coordinates $\boldsymbol{q}=\left(\boldsymbol{p}_{\theta_{2_{1}}}, \boldsymbol{p}_{1_{2}}, \boldsymbol{p}_{\theta_{2_{2}}}, \boldsymbol{p}_{1_{3}}, \boldsymbol{p}_{\theta_{2_{3}}}, \boldsymbol{p}_{1_{4}}, \boldsymbol{p}_{\theta_{2_{4}}}, \boldsymbol{p}_{2_{5}}\right)$ are given by:

$$
\begin{aligned}
& \int_{0}^{\Delta D} \boldsymbol{B}_{\theta_{2_{1}}}^{T} \mathcal{F}_{i_{2}} d X=\boldsymbol{J}_{\theta_{2_{1}}}^{T}(\Delta D)\left(\int_{D_{2}}^{L_{2_{s}}} \operatorname{ad}_{\boldsymbol{\xi}_{1}}^{*} \operatorname{Ad}_{\boldsymbol{g}_{\theta_{2_{s}}}}^{*} \mathcal{F}_{i_{2}} d X+\right. \\
& \left.\int_{L_{2_{s}}}^{\Delta D+L_{1}} \operatorname{ad}_{\boldsymbol{\xi}_{1}}^{*} \operatorname{Ad}_{\boldsymbol{g}_{\theta_{2}}}^{*} \mathcal{F}_{i_{2}} d X\right) \\
& \int_{\Delta D}^{D_{2}} \boldsymbol{B}_{1_{2}}^{T}\left(\mathcal{F}_{i_{1}}+\mathcal{F}_{i_{2}}\right) d X=\mathbf{0} \\
& \int_{\Delta D}^{D_{2}} \boldsymbol{B}_{\theta_{2_{2}}}^{T} \mathcal{F}_{i_{2}} d X=\boldsymbol{J}_{\theta_{2_{2}}}^{T}\left(D_{2}\right)\left(\int_{D_{2}}^{L_{2_{s}}} \operatorname{ad}_{\boldsymbol{\xi}_{1}}^{*} \operatorname{Ad}_{\boldsymbol{g}_{\theta_{2}}}^{*} \mathcal{F}_{i_{2}} d X+\right. \\
& \left.\int_{L_{2 s}}^{\Delta D+L_{1}} \operatorname{ad}_{\boldsymbol{\xi}_{1}}^{*} \operatorname{Ad}_{\boldsymbol{g}_{\theta_{2 s}}}^{*} \mathcal{F}_{i_{2}} d X\right) \\
& \int_{D_{2}}^{L_{2_{s}}} \boldsymbol{B}_{1_{3}}^{T}\left(\mathcal{F}_{i_{1}}+\operatorname{Ad}_{\boldsymbol{g}_{\theta_{2 s}}}^{*} \mathcal{F}_{i_{2}}\right) d X=\mathbf{0} \\
& \int_{D_{2}}^{L_{2_{s}}} \boldsymbol{B}_{\theta_{2_{3}}}^{T} \mathcal{F}_{i_{2}} d X=\int_{D_{2}}^{L_{2_{s}}} \boldsymbol{J}_{\theta_{2_{3}}}^{T} \operatorname{ad}_{\boldsymbol{\xi}_{1}}^{*} \operatorname{Ad}_{\boldsymbol{g}_{\theta_{2_{s}}}}^{*} \mathcal{F}_{i_{2}} d X+ \\
& \boldsymbol{J}_{\theta_{2_{3}}}^{T}\left(L_{2_{s}}\right)\left(\int_{L_{2_{s}}}^{L_{2}} \operatorname{ad}_{\boldsymbol{\xi}_{1}}^{*} \operatorname{Ad}_{\boldsymbol{g}_{\theta_{s}}}^{*} \mathcal{F}_{i_{2}} d X\right) \\
& \int_{L_{2_{s}}}^{\Delta D+L_{1}} \boldsymbol{B}_{1_{4}}^{T}\left(\mathcal{F}_{i_{1}}+\operatorname{Ad}_{\boldsymbol{g}_{\theta_{2 s}}}^{*} \mathcal{F}_{i_{2}}\right) d X=\mathbf{0} \\
& \int_{L_{2_{s}}}^{\Delta D+L_{1}} \boldsymbol{B}_{\theta_{2_{4}}}^{T} \mathcal{F}_{i_{2}} d X=\int_{L_{2_{s}}}^{\Delta D+L_{1}} \boldsymbol{J}_{\theta_{2_{4}}}^{T} \operatorname{ad}_{\boldsymbol{\xi}_{1}}^{*} \operatorname{Ad}_{\boldsymbol{g}_{\theta_{2_{s}}}}^{*} \mathcal{F}_{i_{2}} d X \\
& \int_{\Delta D+L_{1}}^{L_{2}} \boldsymbol{B}_{2_{5}}^{T} \mathcal{F}_{i_{2}} d X=\mathbf{0} \text {. }
\end{aligned}
$$

In developing the equilibrium equation (35), we have used the identity $\boldsymbol{J}_{\theta}^{T} \operatorname{ad}_{\xi}^{*} \mathcal{F}_{i}=\mathbf{0}$ for the straight portion that precedes the insertion orifice. Furthermore, note that $\boldsymbol{B}_{1_{2}}$ has only torsional components, and $\boldsymbol{B}_{\theta_{2_{2}}}(X), \boldsymbol{B}_{\theta_{2_{3}}}(X)$ are equal to zero at $X<$ $\Delta D$ and $X<D_{2}$, respectively.

Considering the equilibrium equations for different CTR systems presented so far (26), (33) and (35), we notice a pattern that can be exploited to produce the equilibrium equations for other particular CTR systems, without going through all the mathematical steps.

\section{Comparison with experiments in literature}

The results of the complete system model (35) are compared with experimentally validated simulations available in the literature [21]. Consider two tubes with a straight portion followed by a constant pre-curvature, as shown in Figure 3 , and physical properties reported in Table I. We seek the equilibrium configuration in two sets of insertion and rotation conditions of the inner tube. In the first set, the full overlap 


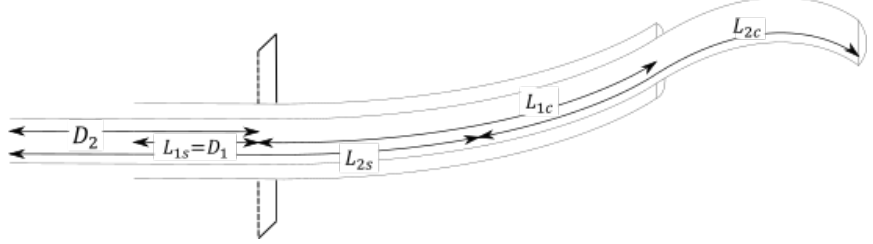

Fig. 3: Sketch of the simulated complete CTR system. Dimensions of the tubes are as follows: straight length $L_{1_{s}}=93.5 \mathrm{~mm}$ (outer) $L_{2_{s}}=218.5 \mathrm{~mm}$ (inner), curved length $L_{1_{c}}=92.3 \mathrm{~mm}$ (outer) $L_{2_{c}}=85 \mathrm{~mm}$ (inner)

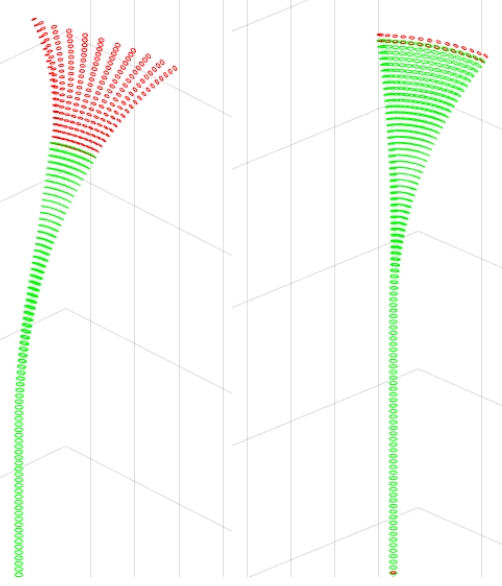

Fig. 4: Equilibrium configurations computed with the variable-strain model for two concentric tubes with straight portion and constant pre-curvature. The inner tube is rotated from $0^{\circ}$ to $280^{\circ}$ in $20^{\circ}$ increments (left) and from $0^{\circ}$ to $200^{\circ}$ in $20^{\circ}$ increments (right). The result of the variable-strain model match well with the one of the standard CTR model [21].

case, $D_{2}=208.5 \mathrm{~mm}$, and $\alpha_{2}$ goes from $0^{\circ}$ to $280^{\circ}$ in $20^{\circ}$ increments. In the second set, the partial overlap case, $D_{2}=170.5 \mathrm{~mm}$, and $\alpha_{2}$ goes from $0^{\circ}$ to $200^{\circ}$ in $20^{\circ}$ increments. $D_{1}$ and $\alpha_{1}$ are always equal to $93.5 \mathrm{~mm}$ and 0 , respectively. We chose a quadratic basis for all the strains involved, except for the last section, where we use $\mathbf{k}_{2}^{*}$.

Figure 4 illustrates the equilibrium configurations obtained with the variable-strain model. A visual comparison shows a good match with the standard CTR model (Fig. 9 of [21]).

\section{Benefits of the PVS model for CTR}

The proposed piecewise variable-strain approach for concentric tube robots presents several benefits. It provides the equilibrium equations as a minimum set of closed-form algebraic equations. The system is easy to handle for control and design optimization since all the boundary and continuity conditions are already intrinsically embedded in the equilibrium equations. The model presents a repetitive pattern that facilitates the scaling to a higher number of tubes. Furthermore, extension to dynamics can be done without any change to the number and type of generalized coordinates. Finally, the PVS model is well-posed to accommodate additional DOFs and incorporate design variation of CTRs as well as generalization to other rod-driven soft robots. In the next section, we include the tubes' insertion and rotation motion as generalized coordinates of the system for the first time.

\section{SLIDING CTR MODEL}

According to the authors' understanding of the standard approach, the tubes' insertion and rotation motion modeling are yet to be fully accomplished [4], [5]. The rotation motion is included as kinematic boundary conditions. Thus, no equations of motion have been produced for these DoFs, which could be used to compute the required actuation torques. Furthermore, the insertion motion appears only in the definition of each overlapping section's domain, which fixes the shared material abscissa between the overlapping tubes once for all. Doing so, the standard CTR model cannot account for time-varying insertion policies if not blending static snapshots using a derivative propagation approach [22]. Accordingly, the recent extension of the standard model to dynamics assumes zero insertion velocity and acceleration [7]. This section will extend the proposed PVS model for CTR to include the input motions as generalized variables and overcome the standard model's above limitations.

Before that, we establish a general result for the differential kinematics of rod's sections with a variable domain. Consider a rod section with material abscissa $X \in[a(t), b(t)]$ and $\boldsymbol{g}(a)=$ $\boldsymbol{I}$. The tip of the section $\boldsymbol{g}(b)$ can be formally represented by the integration of the linear time-varying differential equation (4) through its state-transition matrix $\boldsymbol{g}(b)=\Phi(b, a)$. Then, the derivative of $\boldsymbol{g}(b)$ with respect to time has to include the variation of the domain boundaries in addition to the variation of the shape. This can be calculated as follows.

$$
\frac{\partial \Phi(b, a)}{\partial a} \frac{\mathrm{d} a}{\mathrm{~d} t}=-\widehat{\boldsymbol{\xi}}(a) \boldsymbol{g}(b) \dot{a}, \text { and } \frac{\partial \Phi(b, a)}{\partial b} \frac{\mathrm{d} b}{\mathrm{~d} t}=\boldsymbol{g}(b) \widehat{\boldsymbol{\xi}}(b) \dot{b},
$$

where the first can be considered a "growing from the base" term and the second a "growing from the tip" term. Note that internal cross-sections $\boldsymbol{g}(X)$ will vary only due to the proximal boundary $a$, while they are indifferent to the distal boundary $b$. Finally, the total velocity twist becomes:

$$
\boldsymbol{\eta}(b)=\operatorname{Ad}_{\boldsymbol{g}(b)}^{-1} \int_{a}^{b} \operatorname{Ad}_{\boldsymbol{g}} \dot{\boldsymbol{\xi}} d X+\boldsymbol{\xi}(b) \dot{b}-\operatorname{Ad}_{\boldsymbol{g}(b)}^{-1} \boldsymbol{\xi}(a) \dot{a},
$$

Observe the differences with equation (7) and (10) used so far.

To keep track of the potentially variable boundaries, in the following, we replace the notation $\boldsymbol{g}(b)$ with $\boldsymbol{g}([a, b])$.

\section{A. Complete CTR system with actuation forces and torques}

Let us consider, without loss of generality, two nonoverlapping concentric tubes constrained to be straight before the insertion orifice, as shown in Figure 3. We removed the reference strain discontinuity here for simplicity, reducing the number of required sections to four. First, we define two material abscissas respectively for the outer tube $X_{1}=\left[0, L_{1}\right]$, and the inner tube $X_{2}=\left[0, L_{2}\right]$. Overlapping cross-sections are then related by:

$$
X_{1}=X_{2}-\Delta D,
$$

where $D_{1}$ and $D_{2}$ are generalized coordinates now. In this example, the full set of generalized coordinates is $\boldsymbol{q}=$ $\left(D_{1}, D_{2}, \alpha_{1}, \alpha_{2}, \boldsymbol{p}_{\theta_{2_{1}}}, \boldsymbol{p}_{1_{2}}, \boldsymbol{p}_{\theta_{2_{2}}}, \boldsymbol{p}_{1_{3}}, \boldsymbol{p}_{\theta_{2_{3}}}, \boldsymbol{p}_{2_{4}}\right)$.

Contrary to section III-C, we fix the spatial frame on the insertion orifice. Then, the tubes' sections' kinematics can be 
expressed with equation (32) after being pre-multiplied by a translational homogeneous matrix $\boldsymbol{g}_{t}$, which depends on the insertions $D_{1}$ and $D_{2}$. Let us focus on the overlapping section immediately after the insertion orifice. The kinematics can be written as:

$$
\begin{gathered}
\boldsymbol{g}_{1_{s}}\left(X_{1} \geq D_{1}\right)=\boldsymbol{g}_{t}\left(\left[0,-D_{1}\right]\right) \boldsymbol{g}_{1_{2}}\left(\left[0, D_{1}\right]\right) \boldsymbol{g}_{1_{3}}\left(\left[D_{1}, X_{1}\right]\right) \\
\boldsymbol{g}_{2_{s}}\left(X_{2} \geq D_{2}\right)=\boldsymbol{g}_{t}\left(\left[0,-D_{1}\right]\right) \boldsymbol{g}_{1_{2}}\left(\left[0, D_{1}\right]\right) \boldsymbol{g}_{1_{3}}\left(\left[D_{1}, X_{1}(\Delta D)\right]\right) \\
\boldsymbol{g}_{\theta_{2_{1}}}([0, \Delta D]) \boldsymbol{g}_{\theta_{2_{2}}}\left(\left[\Delta D, D_{2}\right]\right) \boldsymbol{g}_{\theta_{2_{3}}}\left(\left[D_{2}, X_{2}\right], X_{1}(\Delta D)\right),
\end{gathered}
$$

where, for the inner tube, $X_{1}$ is given by (38). The last term $\boldsymbol{g}_{\theta_{23}}\left(\left[D_{2}, X_{2}\right], X_{1}(\Delta D)\right)$ indicates the relative rotation between $X_{1}(\Delta D)$ of the outer tube and $X_{2}$ of the inner tube accumulated in the last section starting from $D_{2}$.

Using the general formula (37) and the discretizations (19), and (20), we obtain the differential kinematics of the section.

$$
\begin{aligned}
& \boldsymbol{\eta}_{1_{s}}\left(X_{1}\right)= \operatorname{Ad}_{\boldsymbol{g}_{1_{3}}\left(X_{1}\right)}^{-1}\left[\boldsymbol{B}_{\alpha} \dot{\alpha}_{1}-\overline{\boldsymbol{\xi}}_{k_{1}}\left(D_{1}\right) \dot{D}_{1}+\boldsymbol{S}_{1_{2}}\left(D_{1}\right) \dot{\boldsymbol{p}}_{1_{2}}\right]+ \\
& \boldsymbol{J}_{1_{3}}\left(X_{1}\right) \dot{\boldsymbol{p}}_{1_{3}} \\
& \boldsymbol{\eta}_{2_{s}}\left(X_{2}\right)= \operatorname{Ad}_{\boldsymbol{g}_{\theta_{2_{s}}}\left(X_{2}\right)}^{-1} \boldsymbol{\eta}_{1_{s}}\left(X_{1}\right)+\boldsymbol{B}_{\alpha} \dot{\alpha}_{2}+\boldsymbol{J}_{\theta_{2_{1}}}(\Delta D) \dot{\boldsymbol{p}}_{\theta_{2_{1}}}+ \\
& \boldsymbol{J}_{\theta_{2_{2}}}\left(D_{2}\right) \dot{\boldsymbol{p}}_{\theta_{2_{2}}}+\boldsymbol{J}_{\theta_{2_{3}}}\left(X_{2}\right) \dot{\boldsymbol{p}}_{\theta_{2_{3}}}-\operatorname{Ad}_{\boldsymbol{g}_{\theta_{2_{s}}}\left(X_{2}\right)}^{-1} \overline{\boldsymbol{\xi}}_{k_{1}}\left(X_{1}\right) \Delta \dot{D}
\end{aligned}
$$

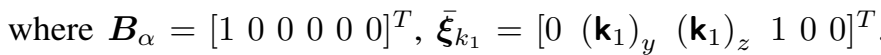
Note that the variable upper boundary of $\boldsymbol{g}_{\theta_{23}}$ is due to $X_{1}(\Delta D)$. Thus, its variation has to be taken with $X_{2}$ fixed.

Equation (40) provides the additional Jacobians corresponding to the input motions. Similar equations can be obtained for the other three sections. Projecting the differential equation (13) by d'Alembert's principle using these additional Jacobians yields the equilibrium equations for the insertion and rotation input forces $\tau_{D_{1}}, \tau_{D_{2}}$, and torques $\tau_{\alpha_{1}}, \tau_{\alpha_{2}}$.

$$
\begin{aligned}
\tau_{D_{1}}= & E J_{1}\left[\boldsymbol{\xi}_{k_{1}}^{T}\left(\boldsymbol{\xi}_{k_{1}}-\boldsymbol{\xi}_{k_{1}}^{*}\right)\left(D_{1}^{+}\right)\right]+E J_{2}\left[\frac{1}{2} \boldsymbol{\xi}_{k_{1}}^{T} \boldsymbol{\xi}_{k_{1}}\left(D_{2}^{+}\right)+\right. \\
& \int_{D_{2}}^{L_{1}+\Delta D}\left(\boldsymbol{\xi}_{k_{1}}^{\prime}+\operatorname{ad}_{\boldsymbol{\xi}_{1}} \overline{\boldsymbol{\xi}}_{k_{1}}\right)^{T} \operatorname{Ad}_{\boldsymbol{g}_{\theta_{2}}} \boldsymbol{\xi}_{k_{2}}^{*} d X+ \\
& \boldsymbol{\xi}_{k_{1}}^{T}\left(\frac{1}{2} \boldsymbol{\xi}_{k_{1}}-\operatorname{Ad}_{\boldsymbol{g}_{\theta_{2}}} \boldsymbol{\xi}_{k_{2}}^{*}\right)\left(L_{1}+\Delta D^{-}\right)- \\
& \left.\boldsymbol{\xi}_{k_{2}}^{T}\left(\boldsymbol{\xi}_{k_{2}}-\boldsymbol{\xi}_{k_{2}}^{*}\right)\left(L_{1}+\Delta D^{+}\right)\right] \\
\tau_{D_{2}}= & E J_{2}\left[\boldsymbol{\xi}_{k_{1}}^{T}\left(\frac{1}{2} \boldsymbol{\xi}_{k_{1}}-\operatorname{Ad}_{\boldsymbol{g}_{\theta_{2}}} \boldsymbol{\xi}_{k_{2}}^{*}\right)\left(D_{2}^{+}\right)-\right. \\
& \int_{D_{2}}^{L_{1}+\Delta D}\left(\boldsymbol{\xi}_{k_{1}}^{\prime}+\operatorname{ad}_{\boldsymbol{\xi}_{1}} \overline{\boldsymbol{\xi}}_{k_{1}}\right)^{T} \operatorname{Ad}_{\boldsymbol{g}_{\theta_{2}}} \boldsymbol{\xi}_{k_{2}}^{*} d X- \\
& \boldsymbol{\xi}_{k_{1}}^{T}\left(\frac{1}{2} \boldsymbol{\xi}_{k_{1}}-\operatorname{Ad}_{\boldsymbol{g}_{\theta_{2}}} \boldsymbol{\xi}_{k_{2}}^{*}\right)\left(L_{1}+\Delta D^{-}\right)+ \\
& \left.\boldsymbol{\xi}_{k_{2}}^{T}\left(\boldsymbol{\xi}_{k_{2}}-\boldsymbol{\xi}_{k_{2}}^{*}\right)\left(L_{1}+\Delta D^{+}\right)\right] \\
\tau_{\alpha_{1}}= & -\tau_{\alpha_{2}} \\
\tau_{\alpha_{2}}= & -\boldsymbol{B}_{\alpha}^{T} \int_{D_{2}}^{L_{1}+\Delta D} \operatorname{ad}_{\boldsymbol{\xi}_{1}}^{*} \operatorname{Ad}_{\boldsymbol{g}_{\theta_{2}}}^{*} \mathcal{F}_{i_{2}} d X \\
&
\end{aligned}
$$

where $\boldsymbol{\xi}_{k}=\left[\begin{array}{llllll}0 & (\mathbf{k})_{y} & (\mathbf{k})_{z} & 0 & 0 & 0\end{array}\right]^{T}$. To obtain equation (41) we
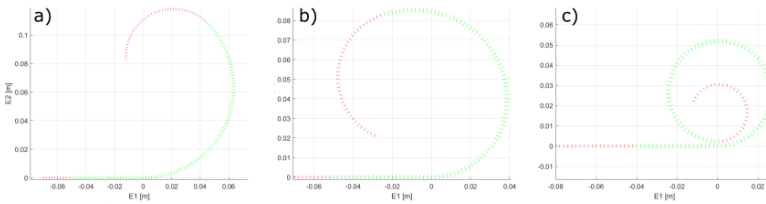

d)

e)

f)

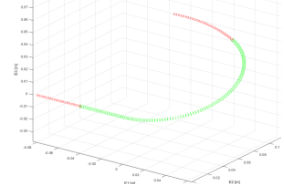

Fig. 5: Equilibrium configurations of two concentric tubes for investigation of the actuation inputs. The top row shows planar configurations while the bottom row resents out-of-plane conformations. The details of the reference shapes and calculated actuation inputs are reported for each cases in Table II.

have also used the following boundary conditions.

$$
\begin{array}{ll}
\overline{\boldsymbol{\xi}}^{T} \mathcal{F}_{i_{1}}(0)=-\tau_{D_{1}}, & \boldsymbol{B}_{\alpha}^{T} \mathcal{F}_{i_{1}}(0)=-\tau_{\alpha_{1}} \\
\overline{\boldsymbol{\xi}}^{T} \mathcal{F}_{i_{2}}(0)=-\tau_{D_{2}}, & \boldsymbol{B}_{\alpha}^{T} \mathcal{F}_{i_{2}}(0)=-\tau_{\alpha_{2}}
\end{array}
$$

According to the Newton law of conservation of momentum, the input forces' and torques' sums have to be zero. It is an excellent verification to check if equation (41) satisfies these conditions. For the input torques, the third equation of (41) ensures that the actuation torques are equal and opposite. For what concerns the input forces, we have:

$$
\begin{aligned}
\tau_{D_{1}}+\tau_{D_{2}}= & E J_{1}\left[\boldsymbol{\xi}_{k_{1}}^{T}\left(\boldsymbol{\xi}_{k_{1}}-\boldsymbol{\xi}_{k_{1}}^{*}\right)\left(D_{1}^{+}\right)\right]+ \\
& E J_{2}\left[\boldsymbol{\xi}_{k_{1}}^{T}\left(\boldsymbol{\xi}_{k_{1}}-\operatorname{Ad}_{\boldsymbol{g}_{\theta_{2}}} \boldsymbol{\xi}_{k_{2}}^{*}\right)\left(D_{2}^{+}\right)\right], \\
\tau_{D_{1}}+\tau_{D_{2}}= & \boldsymbol{\xi}_{k_{1}}^{T}\left(\mathcal{F}_{i_{1}}\left(D_{1}^{+}\right)+\operatorname{Ad}_{\boldsymbol{g}_{\theta_{2}}}^{*} \mathcal{F}_{i_{2}}\left(D_{2}^{+}\right)\right)=0
\end{aligned}
$$

where the last identity, which states that the internal stress is null on the cross-section immediately after the orifice, is justified by the fact that no external forces act on the system from that point onward. Thus, also the input forces are equal and opposite as required.

\section{B. Simulation tests}

Some simulation tests are presented in this section to explore the behavior of the actuation forces and torques. We consider six configurations (three planar and three out-of-plane) of two concentric tubes with physical properties as for Table I. In all the cases, we consider linear non-homogeneous (or constant) pre-curvatures. The linear and non-homogeneous pre-curvature coefficients are varied to study the effect on the actuation forces and torques. In the out-of-plane cases, the inner tube is rotated by $90^{\circ}$ with respect to the outer tube. Figure 5 shows the six configurations studied, while Table II reports the exact values of the non-homogeneous and linear coefficients and the calculated values of the actuation inputs.

The following observations can be made. For case a), the insertion motions $D_{1}$ and $D_{2}$ do not influence the actuation inputs value. In case b), while the insertion of the inner tube $D_{2}$ is still irrelevant, the force input absolute value $\left|\tau_{D}\right|$ increase with the outer tube's insertion and curvature. In c), the force input absolute value $\left|\tau_{D}\right|$ increase with the insertion of the outer tube and the retraction of the inner tube. For the out-of-plane cases, in general, the absolute value $\left|\tau_{D}\right|$ increases with the 
TABLE II: Sliding-rod test results

\begin{tabular}{cccccccc}
\hline & & \multicolumn{2}{c}{ Outer tube } & \multicolumn{2}{c}{ Inner tube } & \multicolumn{2}{c}{ Actuation } \\
\hline & $\alpha_{2}$ & $\begin{array}{c}\text { constant } \\
{[\mathrm{rad} / \mathrm{m}]}\end{array}$ & $\begin{array}{c}\text { linear } \\
{\left[\mathrm{rad} / \mathrm{m}^{2}\right]}\end{array}$ & $\begin{array}{c}\text { constant } \\
{[\mathrm{rad} / \mathrm{m}]}\end{array}$ & $\begin{array}{c}\text { linear } \\
{\left[\mathrm{rad} / \mathrm{m}^{2}\right]}\end{array}$ & $\begin{array}{c}\tau_{D_{2}} \\
{[\mathrm{~N}]}\end{array}$ & $\begin{array}{c}\tau_{\alpha_{2}} \\
{[\mathrm{Nm}]}\end{array}$ \\
\hline a) & $0^{\circ}$ & 10 & 0 & 30 & 0 & 0 & 0 \\
\hline b) & $0^{\circ}$ & 30 & -80 & 30 & 0 & 3.65 & 0 \\
\hline c) & $0^{\circ}$ & 30 & 0 & 50 & 80 & 6.71 & 0 \\
\hline d) & $90^{\circ}$ & 20 & 0 & 10 & 0 & 0.28 & 0.118 \\
\hline e) & $90^{\circ}$ & 20 & 40 & 10 & 0 & -1.45 & 0.122 \\
\hline f) & $90^{\circ}$ & 20 & 0 & 10 & 40 & 1.89 & 0.129 \\
\hline & & & & & & &
\end{tabular}

rotation angle $\alpha_{2}$ up to $90^{\circ}$, and it is symmetric with respect to clockwise or counterclockwise rotations. In particular, the linear coefficients of e) and f) have an opposite contribution to the actuation force $\tau_{D_{2}}$. In case e), $\tau_{D_{2}}$ decreases with the increment of the linear coefficient until it becomes negative, as reported in Table II. In case f), the opposite applies.

\section{Benefits of the sliding-rod PVS model for CTR}

The sliding-rod PVS model presented here proposes a growing (non-material) approach, which allows extending the PVS model to include the tubes' sliding motion without the need of calculating the unknown interaction forces. This new model can be used to control the CTR motion through the actuation force and torques instead of the insertion and rotation kinematics. Torque-controlled CTR can provide a new way to enhance elastic stability and improve interaction forces' control at the end-effector, currently a major concern in the design of CTR [23]. In the present form, the base force equations (41) can also be used to improve fast kinematic controllers of [24], which is based on actuation load sensing.

\section{CONCLUSIONS}

In this paper, the recently proposed piecewise variablestrain approach for modeling highly deformable rods has been adjusted and applied to the case of concentric tube robots. The performances of the new approach have been compared with analytic, simulated, and experimental data available in the literature. Furthermore, the PVS approach has been further extended to include the tubes' insertion motion for the first time, which opens new unexplored possibilities for controlling these kinds of systems.

Future works include experimental validations of the slidingrod PVS model, the addition of external forces, and the extension to dynamics.

\section{APPENDIX A}

\section{ADJOINT REPRESENTATIONS}

$$
\begin{aligned}
\operatorname{Ad}_{\boldsymbol{g}} & =\left(\begin{array}{cc}
\boldsymbol{R} & \mathbf{0}_{3 \times 3} \\
\widetilde{\mathbf{u}} \boldsymbol{R} & \boldsymbol{R}
\end{array}\right), \operatorname{Ad}_{\boldsymbol{g}}^{*}=\left(\begin{array}{cc}
\boldsymbol{R} & \widetilde{\mathbf{u}} \boldsymbol{R} \\
\mathbf{0}_{3 \times 3} & \boldsymbol{R}
\end{array}\right), \\
\operatorname{ad}_{\boldsymbol{\xi}, \boldsymbol{\eta}} & =\left(\begin{array}{cc}
\widetilde{\mathbf{k}}, \widetilde{\mathbf{w}} & \mathbf{0}_{3 \times 3} \\
\widetilde{\mathbf{u}}, \widetilde{\mathbf{v}} & \widetilde{\mathbf{k}}, \widetilde{\mathbf{w}}
\end{array}\right), \operatorname{ad}_{\boldsymbol{\xi}, \boldsymbol{\eta}}^{*}=\left(\begin{array}{cc}
\widetilde{\mathbf{k}}, \widetilde{\mathbf{w}} & \widetilde{\mathbf{u}}, \widetilde{\mathbf{v}} \\
\mathbf{0}_{3 \times 3} & \widetilde{\mathbf{k}}, \widetilde{\mathbf{w}}
\end{array}\right)
\end{aligned}
$$

\section{REFERENCES}

[1] J. Burgner-Kahrs, D. C. Rucker, and H. Choset. Continuum robots for medical applications: A survey. IEEE Transactions on Robotics, 31(6):1261-1280, 2015.
[2] Arthur W. Mahoney, Hunter B. Gilbert, and Robert J. Webster III. A review of concentric tube robots: modeling, control, design, planning, and sensing, chapter Chapter 7, pages 181-202. 2016.

[3] H. Alfalahi, F. Renda, and C. Stefanini. Concentric tube robots for minimally invasive surgery: Current applications and future opportunities. IEEE Transactions on Medical Robotics and Bionics, 2(3):410-424, 2020.

[4] P. E. Dupont, J. Lock, B. Itkowitz, and E. Butler. Design and control of concentric-tube robots. IEEE Transactions on Robotics, 26(2):209-225, 2010.

[5] D. C. Rucker, B. A. Jones, and R. J. Webster III. A geometrically exact model for externally loaded concentric-tube continuum robots. IEEE Transactions on Robotics, 26(5):769-780, 2010.

[6] J. Ha, G. Fagogenis, and P. E. Dupont. Modeling tube clearance and bounding the effect of friction in concentric tube robot kinematics. IEEE Transactions on Robotics, 35(2):353-370, 2019.

[7] J. Till, V. Aloi, K. E. Riojas, P. L. Anderson, R. J. Webster III, and C. Rucker. A dynamic model for concentric tube robots. IEEE Transactions on Robotics, pages 1-15, 2020.

[8] Hunter B. Gilbert, D. Caleb Rucker, and Robert J. Webster III. Concentric Tube Robots: The State of the Art and Future Directions, pages 253-269. Springer International Publishing, Cham, 2016.

[9] F. Renda, C. Armanini, V. Lebastard, F. Candelier, and F. Boyer. A geometric variable-strain approach for static modeling of soft manipulators with tendon and fluidic actuation. IEEE Robotics and Automation Letters, 5(3):4006-4013, 2020.

[10] F. Boyer, V. Lebastard, F. Candelier, and F. Renda. Dynamics of continuum and soft robots: a strain parametrization based approach. soumis à IEEE TRO, October 2019.

[11] R.M. Murray, Z. Li, and S.S. Sastry. A Mathematical Introduction to Robotic Manipulation. Taylor \& Francis, Boca Raton, USA, 1994.

[12] F. Renda and L. Seneviratne. A geometric and unified approach for modeling soft-rigid multi-body systems with lumped and distributed degrees of freedom. In 2018 IEEE International Conference on Robotics and Automation (ICRA), pages 1567-1574, May 2018.

[13] G. F. Carrier. The spaghetti problem. The American Mathematical Monthly, 56(10):669-672, 1949.

[14] G. Cicconofri and A. DeSimone. A study of snake-like locomotion through the analysis of a flexible robot model. Proceedings of the Royal Society A: Mathematical, Physical and Engineering Sciences, 471(2184):20150054, 2015

[15] C. Armanini, F. Dal Corso, D. Misseroni, and D. Bigoni. Configurational forces and nonlinear structural dynamics. Journal of the Mechanics and Physics of Solids, 130:82 - 100, 2019.

[16] A. Humer, I. Steinbrecher, and L. Vu-Quoc. General sliding-beam formulation: A non-material description for analysis of sliding structures and axially moving beams. Journal of Sound and Vibration, 480:115341, 2020.

[17] F. Boyer and F. Renda. Poincare's equations for cosserat media: Application to shells. Journal of Nonlinear Science, 2016.

[18] P. Sears and P. Dupont. A steerable needle technology using curved concentric tubes. In 2006 IEEE/RSJ International Conference on Intelligent Robots and Systems, pages 2850-2856, 2006.

[19] R. J. Webster, A. M. Okamura, and N. J. Cowan. Toward active cannulas: Miniature snake-like surgical robots. In 2006 IEEE/RSJ International Conference on Intelligent Robots and Systems, pages 2857-2863, 2006.

[20] R. J. Webster, J. M. Romano, and N. J. Cowan. Mechanics of precurvedtube continuum robots. IEEE Transactions on Robotics, 25(1):67-78, 2009.

[21] D.C. Rucker, R. J. Webster III, G. S. Chirikjian, and N. J. Cowan. Equilibrium conformations of concentric-tube continuum robots. The International Journal of Robotics Research, 29(10):1263-1280, 2010.

[22] D. C. Rucker and R. J. Webster. Computing jacobians and compliance matrices for externally loaded continuum robots. In 2011 IEEE International Conference on Robotics and Automation, pages 945-950, 2011.

[23] J. Ha, F. C. Park, and P. E. Dupont. Optimizing tube precurvature to enhance the elastic stability of concentric tube robots. IEEE Transaction on Robotics, 33(1):22-37, 2017.

[24] R. Xu, A. Asadian, A. S. Naidu, and R. V. Patel. Position control of concentric-tube continuum robots using a modified jacobian-based approach. In 2013 IEEE International Conference on Robotics and Automation, pages 5813-5818, 2013. 